\title{
Comparison of the Low Power Wireless Technologies for Smart Refuelling
}

\author{
Sneha Kishore Kumar Rane ${ }^{1}$, Rohini S Hallikar ${ }^{2}$ \\ M. Tech, Communication Systems, RV College of Engineering, Bengaluru ${ }^{1}$ \\ Assistant Professor, RV College of Engineering, Bengaluru ${ }^{2}$
}

\begin{abstract}
Wireless communication is widely used in the field of the smart refueling but making the system ultra-low power is the major concern. In application area like mining and construction site, there is a need to use the nonreplaceable low power devices which have more battery life. This paper discusses about the various technologies that can be employed for the wireless communication of the smart refueling and gives the idea in choosing the right technology. Sub-1GHz technology is used in the $866 \mathrm{MHz}$ (free frequency band in India and has low spectral pollution) with the proper tool and product to build a wireless module for automatic vehicle recognition (AVR) system. This module will use the data read by the RFID reader and transmit it to the wireless receiver at the terminal giving low power and low-cost solution to attain fast and smooth refueling.
\end{abstract}

Keywords: Sub-1GHz; low power; Automatic vehicle recognition (AVR).

\section{INTRODUCTION}

In this era, there is the rapid advancement in the field of wireless communication. With the advanced world, there is a need to use the appropriate advanced technologies to get the seamless connectivity in the field of wireless communication. The increase in demand for the speed and convenience, the various technologies can be used but with this increasing speed there is also increase in the power consumption and cost. Various methods and counter technologies have been used to decrease these parameters.

In the field of fueling the intelligent systems have been incorporated, which increases the speed, convenience and even safety which makes it Smart Refueling system. Automatic vehicle recognition (AVR) helps in the automation of the fueling, giving higher vehicle efficiency for the filling station. The arrangement is easy to install and is low cost these can be used in the application like mining and construction area. The idea is to use the low power technology and ultralow power circuit for attaining the seamless wireless communication.

To develop the AVR solution, there is a need of RF frontend that helps to have the efficient communication between the devices present in the application area by developing the device that operates in $866 \mathrm{MHz}$ which is free band in India. The application area can be the remote place and hence there is a need to develop a product that is battery operated and which is also non-replaceable, the solutions presently have the wireless modules, microcontrollers and other devices, and the power consumption of this is optimum. But now to develop new product, the ultra-low power consumption solutions are considered, so that the battery life of the device can increase or extend up to two to three years. And hence there is a need to understand and use all the low power operated solutions in each stage of the development. In this application, normally the distance will be short range of around 50 to 70 meters, so assuming this distance the survey is conducted.

\section{TECHNOLOGY FOR RF FRONTEND}

We need to select features in such a way that it is widely used and has good future scope. The first step is to choose the right technology for the communication considering the efficiency, cost, area of application, penetration power, range and the power consumption. Co-existence or spectral pollution must be avoided, this can be done by using the frequency bands that are not highly used like frequency band under $5 \mathrm{GHz}$. The technology used should be such that it should be compatible with the other legacy devices. There will be also the possibility that the external antenna used has a lengthy co-axial cable, so there is a need to take care of the attenuation.

A. Selecting the technology:

To build an advanced and smart wireless system, mostly the two frequency bands of industrial, scientific, and medical (ISM) radio band options that are $2.4 \mathrm{GHz}$ or sub- $\mathrm{GHz}$ frequencies will be chosen. By pairing one or other according to the requirement can provide the best combination of the wireless performance and also the economy [5]. 
The priorities are the Range, Power consumption, Antenna size, Data rates, Interoperability (standards). The technologies like Wi-Fi® ${ }^{\circledR}$, Bluetooth ${ }^{\circledR}$ and ZigBee ${ }^{\circledR}$ are mostly used in today's markets [17]. However, for low-datarate and low power applications, like home security, home automation, smart metering or in automatic vehicle recognition (AVR), sub-GHz wireless systems offer several advantages, including longer range, reduced power consumption and less deployment and operating costs.

In India, the free frequency band for low power wireless equipment available are

- Indoor use of lower power wireless equipment at $2.4-2.4835 \mathrm{Ghz}$.

- Indoor use of lower power wireless equipment at $5 \mathrm{Ghz}$.

- outdoor use of lower power wireless equipment at 5.825-5.875Ghz.

- Use of lower power wireless equipment at $865-867 \mathrm{Mhz}$

- Low power control of cranes $335 \mathrm{Mhz}$

- Use of low power equipment citizen band from 26.975-27.383Mhz

So, the attempt must be made to operate the device in this available bandwidth.

B. Techologies and their cons with respect to the application ( all the pros and cons are with respect to required application).

- Bluetooth technology:

Bluetooth technology is used for the devices with high demands for small footprint, low-power consumption and lowcost. Though low power consumption is the advantage, it provides less range of 10 meters. In this application the devices can distant from each other.

- Bluetooth low energy technology:

Bluetooth v4.0 low-energy technology is specially well suited for sensors, actuators and other small devices that requires extremely low power consumption. It can offer high number of nodes with the limited latency and has very short wake-up or the connection time. This will be the efficient solution if the range is less.

- Wireless LAN (Local area network):

It is well suited for monitoring, configuring and data acquiring, but can also be used for time critical control. Range is 200 meter, however; the obstacles and interference could lower the range substantially. The Co-existence also affects and causes interference. And mainly power consumption by the devices will be more.

- 6LoWPAN

6LoWPAN is an acronym of IPv6 over Low Power Wireless Personal Area Networks and it is an attractive technology, since it is IP-based and this will cut down on packet overhead, and will allow more space for the payload data. But this technology is not used full fledge in India and requires IPv6 support modules.

\section{- JenNet-IP}

The JenNet-IP protocol that combines IEEE802.15.4-based wireless network technology and Internet Protocol (IP) to achieve integration between the two domains, providing a solution for the wireless 'Internet of Things'.

This will allow the nodes of a wireless network to be controlled both wirelessly and within the network and from IPconnected device, like smart phone, which may be remotely located.

- Single chip ANTTM ICs/solutions

This is an ideal fit for a wide range of ultra-low power wireless network applications including hubs for sports, wireless sensors and the healthcare products. ANT ${ }^{\mathrm{TM}}$ is a proven, flexible, ultra-low power and easy-to-use network protocol for the $2.4 \mathrm{GHz}$ ISM frequency band communication. It provides multi-vendor interoperability through the ANT+ managed network and member companies of the open ANT+ Alliance.

\section{- ZigBee}

This technology can provide more battery life and Range but as it operates at $2.4 \mathrm{GHz}$ the coexistence can be occurred and also the replacement of the device can also be costly. [ refer Texas instruments].

\section{- XBee}

Digi International XBee Wi-Fi embedded RF modules provide simple serial to IEEE 802.11 connectivity. It provides low power and low cost solutions. It gives developers the fastest IP to device and device to cloud capability. It provides the Over-the-air data rates up to 65Mbps. 


\section{- LoRa}

LoRaWANTM is a Low Power Wide Area Network (LPWAN). Range is more about $15 \mathrm{~km}$ and there are several layers of encryption provides the low power solution with many nodes, but this technology is costly and requires many certifications.

\section{- Sub- $1 \mathrm{GHz}$}

Range is up to $1 \mathrm{~km}$ and no coexistence occurs. Battery life is expected up to 10-20 years. But the antenna size is bigger compared to other technology. If the antenna size is not the constraint this technology gives the better efficiency.

Table 1: Comparison of the ZigBee, SUB-1GHz and LoRa

\begin{tabular}{|c|c|c|c|}
\hline & ZigBee & sub-1 GHz & LoRa \\
\hline Range & $10-100 \mathrm{~m}$ & up to $1 \mathrm{~km}$ & $15 \mathrm{~km}$ \\
\hline Max data rate & $250 \mathrm{Kbps}$ & $1-500 \mathrm{kbps}$ & $0.3-50 \mathrm{kbps}$ \\
\hline Modulation & OQPSK/DSSS & FSK & $\begin{array}{l}\text { FSK, GFSK, LoRa } \\
\text { TECH modulation }\end{array}$ \\
\hline Battery life & $3-5$ years & $10-20$ years & more than 10 years \\
\hline Advantages & $\begin{array}{l}* \text { collision avoidance } \\
* \text { low cost modules } \\
\text { *patent free protocol } \\
* 30 \mathrm{~ms} \text { for device } \\
\text { searching, } 15 \mathrm{~ms} \text { for activation } \\
\text { and } 15 \mathrm{~ms} \text { for channel access } \\
* \text { AES } 128\end{array}$ & $\begin{array}{l}\text { *penetration capability } \\
\text { *bend around an obstacle, } \\
\text { reducing the blocking effect } \\
\text { *co-existence is not a problem } \\
\text { *antenna gain } \\
\text { * No qualification/certification fees } \\
\text { except FCC/ETSI } \\
\text { *Maximum flexibility with } \\
\text { application specific protocol stacks }\end{array}$ & $\begin{array}{l}\text { *security } \\
* \text { Replacement cost } \\
\text { will be high. } \\
\text { *long range }\end{array}$ \\
\hline Disadvantages & $\begin{array}{l}\text { *low complexity, } \\
\text { *less penetration power } \\
\text { compared to sub-1GHz } \\
\text { *coexistence (higher packet } \\
\text { loss and retransmission is } \\
\text { required) }\end{array}$ & $\begin{array}{l}\text { *device designed will not fit } \\
\text { globally } \\
\text { *larger antenna nearly } 2.5 \text { times } \\
\text { larger }\end{array}$ & $\begin{array}{l}\text { * bit costly modules } \\
* \text { certification to } \\
\text { commercialization } \\
\text { *LoRa chips and } \\
\text { module }\end{array}$ \\
\hline
\end{tabular}

The Table 1 shows the comparison of the ZigBee, SUB-1GHz and the LoRa technology; it can be observed that other than antenna size, the SUB-1GHz provides more advantages as provided below.

\section{SUB-1GHZ}

After this literature survey, Sub-1GHz seemed to be the appropriate technology.

Following are the reasons for choosing Sub-1 GHz:

- It has long range.

- Good penetration capability.

The remote areas can be any place like mining area, where there can be obstacles. The data packets sent will be critical and fast, cannot afford loosing it.

- It can bend around an obstacle, reducing the blocking effect.

- The antenna gain in high signals do not fade easily.

- Co-existence is not a problem and hence any Bluetooth operated device will not interfere.

- No qualification or certification fees except FCC/ETSI.

- Maximum flexibility with application specific protocol stacks.

All the feature match the requirement and hence it is most suitable technology in the remote area

And finally, we need to see that the communication should be in the narrow band. Following are the reasons:

- In the construction or mining site where there are many types of equipment that are radio controlled using narrow band will be optimal.

- It will help to attain the long range and also this node will help to communicate with the distant hub directly, without the hopping from every node.

By using the transceiver with the antenna of cable length $3 \mathrm{~m}$ exclusively for $868 \mathrm{MHz}$ frequency, following results are obtained. 


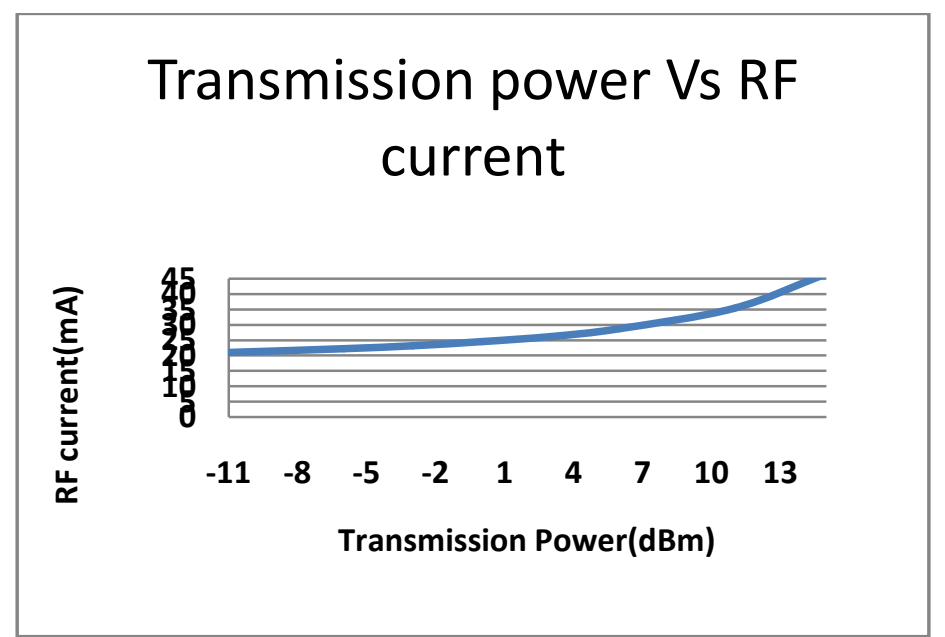

Fig:2 Graph of the RF current Vs transmission power.

From the above graph (Fig 2) it can be observed that even at the transmission power of around $-11 \mathrm{dBm}$ the current used is $21 \mathrm{~mA}$, with the $100 \%$ data transmission at 100 meters. Even with the obstacles the data transmission rate was optimum.

\section{CONCLUSIONS}

The SUB-1GHz can provide the low power solution and the low-cost solution. In area such as mining and construction, where the refuelling is necessary, the devices used are almost primary battery powered and non-replaceable, hence this solution can be effective. As the $866 \mathrm{MHz}$ frequency band is not heavily used, there no coexistence or there is less spectral pollution.

It was also observed that by using the battery of around 5.6Ah capacity, the battery life of around 11days was determined when used continuously. As the signals can penetrate more compared to $2.4 \mathrm{GHz}$ signals, this can used in many remote areas with obstacles.

\section{REFERENCES}

[1] Stefan Aust et al., "Performance evaluation of Sub $1 \mathrm{GHz}$ wireless sensor networks for the smart grid",IEEE 37th Conference on Local Computer Networks (LCN), oct 2012 pp. 866-870

[2] Sayfe Kiaei et.al., "Introduction to the Special Issue on Low Power Wireless Communications" IEEE transactions on circuits and systems - ii: analog and digital signal processing, vol. 44, no. 6, June 1997

[3] Takashi Takeuchi and Takahide Terada, "Evaluation of Wireless Communication Performance in a Li-ion Battery System", Journal of Automation and Control Engineering, Vol. 2, No. 1, March 2014 pp. 26-30.

[4] Sami Akin. "The Interplay Between Data Transmission Power and Transmission Link Utilization” IEEE communications letters, vol. 19, no. 11, November 2015 pp. 1901-1911.

[5] Masoud Babaie et al., "A Fully Integrated Bluetooth Low-Energy Transmitter in 28 nm CMOS With $36 \%$ System Efficiency at 3 dBm" IEEE JOURNAL OF SOLID-STATE CIRCUITS, VOL. 51, NO. 7, JULY 2016, pp. 1547- 1565.

[6] Tianqi Wang et al., "Link Energy Minimization in IR-UWB Based Wireless Networks" ,IEEE transactions on wireless communications, VOL. 9, NO. 9, SEPTEMBER 2010, pp. 70-84.

[7] Bjarte Nystøyl and Torstein Ermesjø, "CC112X/CC120X On-Chip Temperature Sensor” Application Report SWRA415C-June 2014.

[8] Abhishek Chattopadhyay, "Basic RF Testing of CCxxxx Devices", Application Report SWRA370-August 2011.

[9] Matthew Loy et.al," ISM-Band and Short Range Device Antennas”, Application Report, SWRA046A - March 2005 - Revised August 2005

[10] Fredrik Eriksen, "CC112X/CC120X On-Chip Battery Monitor", Application Report, SWRA471-December 2014

[11] “CC112x, CC1175Silicon Errata” Literature Number: SWRZ039D June 2011-Revised April 2015.

[12] Sebastian fudickar. et al., "An Energy Efficient Mobile Device for Assisted Living Applications," IEEE online conference on Green Communications(GreenCom), pp. 133-138, 2012.

[13] Mohammad Ghamari et al., "Comparison of Low-Power Wireless Communication Technologies for Wearable Health-Monitoring Applications ," International Conference on Computer, Communication, and Control Technology, April 21 - 23 in Imperial Kuching Hotel, Kuching, Sarawak, 2015, pp. 1-6.

[14] Konstantinos Domdouzis et al., "WIRELESS SENSOR NETWORKING IN THE CONSTRUCTION INDUSTRY - PROSPECTS AND PROBLEMS", In: Khosrowshahi, F (Ed.), 20th Annual ARCOM Conference, 1-3 September 2004, Heriot Watt University. Association of Researchers in Construction Management, Vol. 2, pp. 1107-20.

[15] Stefan Aust et al., "Sub 1GHz Wireless LAN Deployment Scenarios and Design Implications in Rural Areas", IEEE Globecom 2011 Workshop on Rural Communications-Technologies, Applications, Strategies and Policies (RuralComm 2011) pp. 1045-1049

[16] Shubhangi Bagal et al., "Considerations For Wireless Communication At 868MHz", Annual IEEE India Conference (INDICON), 2014

[17] "Key Priorities for Sub-GHz Wireless Deployment” Silicon Laboratories Inc., Austin, TX

[18] http://www.nxp.com 